\title{
Analysis of Tip Stability in Adhesion Process in AFM Using Potential Energy Surface: Stability Versus Dissipation*
}

\author{
Yasuhiro Senda ${ }^{\dagger}$ \\ Department of Applied Science, Yamaguchi University, Ube, Yamaguchi 755-8611, Japan \\ Janne Blomqvist and Risto Nieminen \\ COMP Centre of Excellence, Department of Applied Physics, \\ Aalto University, P.O. Box 11100, 00076 Aalto, Finland \\ (Received 5 January 2018; Accepted 17 March 2018; Published 9 May 2018)
}

\begin{abstract}
We analyze the stability of the atomic configurations of tips in the adhesion process in noncontact atomic force microscopy (AFM) using a potential energy surface (PES). We calculate the PES for two types of atomic configurations of the tip as typical cases of reconstruction and irreversible change during the adhesion process. The stability of the tips after atomic contact with the surface is explained on the basis of the calculated PESs, which are affected by the strength of the atomic bond between the tip and the surface. It is shown from the computational model for the AFM that an unstable tip leads to a larger energy dissipation compared to that for a stable tip. [DOI: 10.1380/ejssnt.2018.132]
\end{abstract}

Keywords: Computer simulation; Atomic force microscopy; Adhesion; Energy dissipation

\section{INTRODUCTION}

Friction and energy dissipation originate from the atomic interactions at a surface. Dissipation has been observed in noncontact atomic force microscopy (AFM), and a map of the dissipation shows atomic-scale features for several surfaces [1-9]. The origin of dissipation in AFM has been intensively discussed by theoretical and computational methods [10-26]. A stochastic friction mechanism for the dissipation has been proposed [10-14], in which the tip of the cantilever is treated as a Brownian particle fluctuated by random and interaction forces; however, it cannot quantitatively reproduce the dissipation observed in experiments $[13,14]$. To date, the most acceptable mechanism for dissipation is atom adhesion on a surface [15-23]. This adhesion results in hysteresis of the force between the tip and the surface, and this nonconservative force causes energy dissipation in the oscillation of the cantilever, which is comparable to the experimental case.

The stability of the tip apex is a key issue in the adhesion process in AFM. In the adhesive mechanism, the atomic structure of the tip apex remains intact after adhesive atomic contact, and it is reversibly reconstructed during the adhesion process. However, in AFM experiments, the tip apex often experiences an instability; specifically, the atomic structure of the tip apex is irreversibly broken owing to the interaction with the surface [1]. Thus, it is necessary to prepare a stable tip in order to observe the force curves and energy dissipation.

We have investigated the tip stability in the adhesion process using a computational model for AFM, in which the cantilever is described by a spring, and the atomic system of the tip apex and surface is calculated by molecular dynamics [24]. The oscillation of the spring is concurrently coupled with the motions of the atoms using

\footnotetext{
* This paper was presented at the 8th International Symposium on Surface Science, Tsukuba International Congress Center, Tsukuba, Japan, October 22-26, 2017.

† Corresponding Author: senda@yamaguchi-u.ac.jp
}

(a)

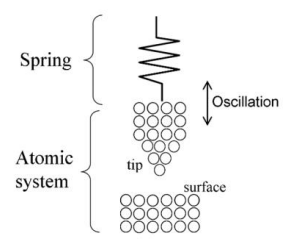

(b)


FIG. 1. (a) Schematic of the model for AFM used in previous studies [24]. The oscillation of the cantilever is described by that of a spring, which is connected to the atomic system of the tip and surface. (b) Two types of ionic structures of the tip used in the atomic system of the AFM model and the time evolutions of the ionic structures upon approach and retraction [24]. The type 1 tip consists of a layer composed of five O (white) ions and four $\mathrm{Mg}$ (black) ions at the surface of the $\mathrm{MgO}$ crystal with a single $\mathrm{Mg}$ ion mounted at the center of this layer. The type 2 tip consists of a layer composed of eight $\mathrm{O}$ ions and eight $\mathrm{Mg}$ ions at the surface of the $\mathrm{MgO}$ crystal with a pair of $\mathrm{Mg}$ and $\mathrm{O}$ ions mounted onto this layer. After a tip approaches the surface, the ionic configuration of the tip is reconstructed for the type 1 tip, whereas it irreversibly collapses for the type 2 tip.

a coupling method. The details of the coupling model for AFM are explained in [25, 26], and a schematic of the model is shown in Fig. 1(a), in which the spring oscillates, and the tip of the atomic system approaches and retracts from the surface. For the atomic model, a tip consisting of $\mathrm{Mg}$ and $\mathrm{O}$ ions with an ionic potential is used and faces a $\mathrm{MgO}$ surface. Dissipation images of the surface of the ionic crystals are demonstrated with atomic reso- 


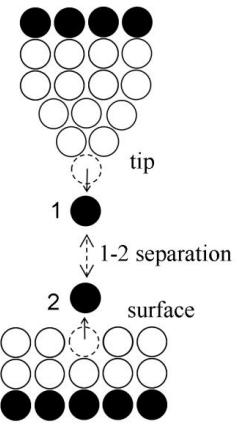

(a)

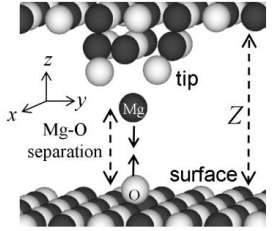

(b)

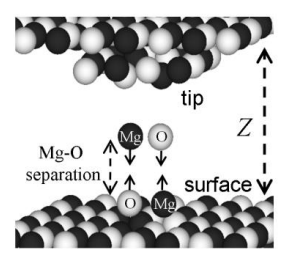

(c)

FIG. 2. (a) Schematic of the constraint applied during energy minimization of the ionic system. The coordination of the ions drawn by solid circles is fixed, whereas the other atoms (open circles) are free during minimization. (b) Snapshot of the ionic system of the type 1 tip. During minimization, the $\mathrm{Mg}$ ion at the apex of the tip and the $\mathrm{O}$ ion of the surface just below the $\mathrm{Mg}$ ion are fixed at some $\mathrm{Mg}-\mathrm{O}$ separation. (c) Snapshot of the system of the type 2 tip. The ion pair of $\mathrm{Mg}$ and $\mathrm{O}$ at the apex of tip and the ion pair of the surface just below the tip are fixed.

lution $[3,4,6]$, and the surface of the $\mathrm{MgO}$ also has been successfully imaged by non-contact AFM [27]. We have shown that the energy dissipation of the spring originates from the adhesion of the ions in the atomic system and have investigated the stability of a few types of $\mathrm{MgO}$ tips during the adhesion process. The ionic configuration of the type 1 tip is reconstructed even after atomic contact with the surface, as shown in Fig. 1(b), whereas that of the type 2 tip is irreversibly changed after contact.

In this study, we investigate the mechanism of the tip stability of these two tip types as typical cases of reconstruction and irreversible change during the adhesion process. We analyze the tip stability using the potential energy surface (PES). Analysis using the PES has explained atomic adhesion and hysteresis on a variety of surfaces $[15,19,21,23]$ and in nanojunctions [28]. We explain not only the adhesion between the tip and the surface but also the stability of the tip during the adhesion process in terms of the calculated PES. Moreover, by comparing the above two types of tip: stable and instable, the dependence of the stability on the types of the tip apex is discussed using the PESs.

\section{METHOD}

A tip consisting of $\mathrm{Mg}$ and $\mathrm{O}$ ions with a classical ionic potential has been shown to be good model for a wide class of polar tips of AFM probes [29]. The pairwise potential comprises parts of the Coulombic, dispersion, and repulsive interactions between ions [30]. The total potential energy of the ionic system is assumed to be composed of the sum of the pair potentials. Thus, the defined potential energy of the ionic $\mathrm{MgO}$ system is minimized with constraints, as shown in Fig. 2(a), in which the coordination of the ions in the bottom layer of the surface and the upper layer of the tip is fixed, and that of the apex ion of the tip (ion 1) and the ion of the surface just below the tip (ion 2) is also fixed. The other ions [open
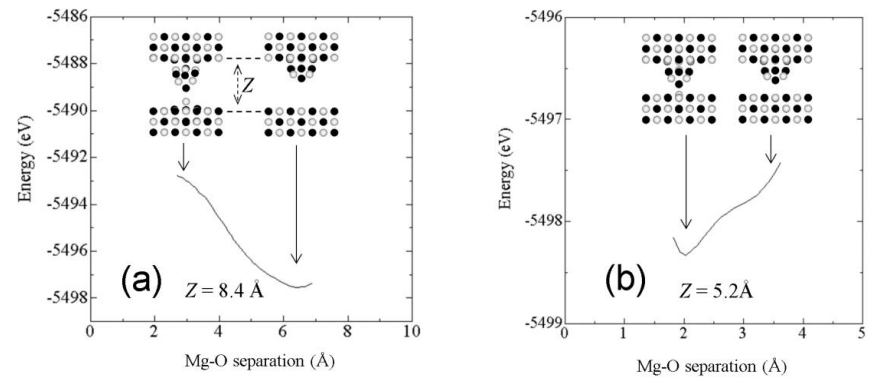

FIG. 3. Calculated potential energy surfaces (PESs) of the ionic system of the type 1 tip as a function of the $\mathrm{Mg}-\mathrm{O}$ separation for (a) $Z=8.4 \AA$ and (b) $Z=5.2 \AA$. The optimized ionic configurations at the corresponding $\mathrm{Mg}-\mathrm{O}$ separations are superimposed.

circles in Fig. 2(a)] are allowed to move and relax during minimization. The optimized configuration of the ionic system of the tip and surface is obtained for the separation between 1 and 2 ions, which is modulated by shifting ions 1 and 2 vertically. Energy minimization is performed at each separation, and the minimized potential energy, the so-called potential energy surface (PES), as a function of the separation is derived.

We apply constraints to the ionic $\mathrm{MgO}$ system of the type 1 and 2 tips, as shown in Fig. 2(b, c), respectively. The periodic boundary condition is applied to the system along the $x$ and $y$ directions. The separation between $\mathrm{Mg}$ and $\mathrm{O}$ ions is modulated, and the PES as a function of the $\mathrm{Mg}-\mathrm{O}$ separation is derived. In the AFM model using the coupling method, the tip of the ionic system approaches and retracts from the surface as shown in Fig. 1, where the height of the tip from the surface $Z$ is changed. We investigate how the PES depends on $Z$.

\section{RESULTS AND DISCUSSION}

\section{A. Type 1 tip: stable tip}

When the tip is far from the surface $(Z=8.4 \AA)$, the calculated PES is minimized when the distance between the $\mathrm{Mg}$ and $\mathrm{O}$ ions is large, as shown in Fig. 3(a). In this case, the $\mathrm{Mg}$ and $\mathrm{O}$ ions energetically prefer to be separated from each other and are located at the original lattice sites of the tip and surface, respectively. In contrast, if the tip is close to the surface $(Z=5.2 \AA)$, the ions are attracted each other, and an ionic bond forms between the $\mathrm{Mg}$ and $\mathrm{O}$ ions, where the minimum of the $\mathrm{PES}$ is achieved when the separation is equal to the $\mathrm{Mg}-\mathrm{O}$ bond length, as shown in Fig. 3(b).

The height dependence of the PES of the ionic system of the type 1 tip is shown in Fig. 4. The minima of the PESs at points 1 and 2 correspond to states in which the $\mathrm{Mg}$ ion of the tip is separated from the $\mathrm{O}$ ion of the surface in the same way as the case of $Z=8.4 \AA$ in Fig. 3(a), whereas points 3 and 4 correspond to the formation of a $\mathrm{Mg}-\mathrm{O}$ ionic bond. When the tip is far from the surface $(Z=6.4 \AA)$, the $\mathrm{Mg}$ ion of tip is separated from the $\mathrm{O}$ ion on the surface (point 1). As the tip approaches the surface to $Z=5.9 \AA$, the $\mathrm{Mg}$ ion is still separated from the $\mathrm{O}$ ion (point 2). However, another minimum (point 

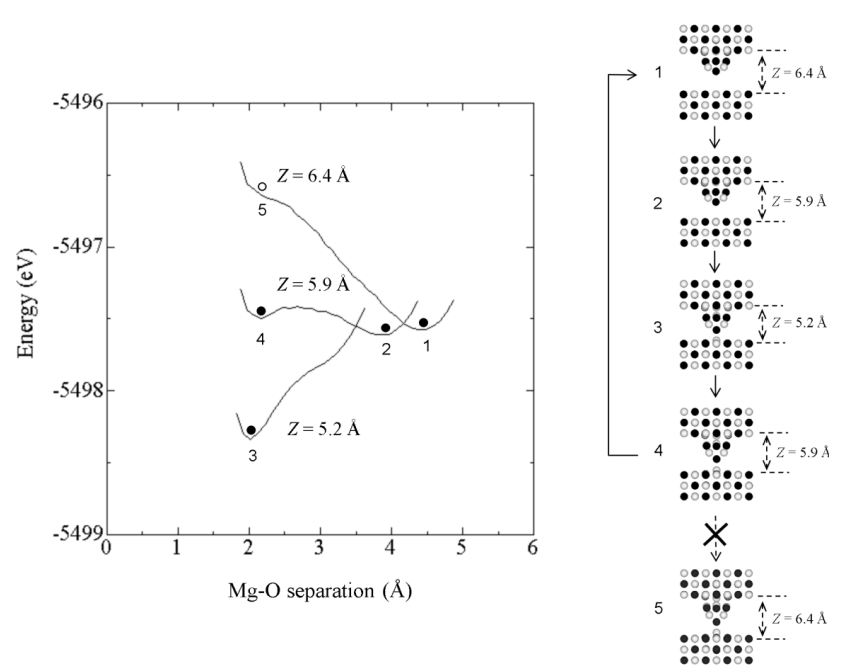

FIG. 4. Height dependence of the PES of the ionic system of the type 1 tip. The minima in the PESs are indicated by the filled circles. The ionic configurations at points $1-5$ are illustrated on the right side.

4) exists on the PES, and it is separated from point 2 by an energetic barrier. As the tip further approaches the surface $(Z=5.2 \AA)$, the minima at points 1 and 2 become shallow, the minimum in the PES changes to point 3, and a $\mathrm{Mg}-\mathrm{O}$ bond forms between the tip and the surface. When the tip retracts from the surface, the $\mathrm{Mg}-\mathrm{O}$ bond still remains up to $Z=5.9 \AA$ (point 4). As retraction continues, the minimum in the PES changes from point 4 to point 1 instead of point 5 , at which the formation of the $\mathrm{Mg}-\mathrm{O}$ bond is energetically unstable. These motions of the $\mathrm{Mg}$ and $\mathrm{O}$ ions during the adhesion process are the same as those observed with the AFM model in Fig. 1(a) and can be explained on the basis of the calculated PESs.

The hysteresis of the ionic system originates from the double minimum points accompanying the energetic barrier on the PES at $Z=5.9 \AA$. Points 2 and 4 exist on the PES, and the ionic system cannot simply transfer between these points owing to the considerable large barrier between them. The $\mathrm{Mg}$ ion is separated from the $\mathrm{O}$ ion when the tip approaches the surface (point 2), whereas a $\mathrm{Mg}-\mathrm{O}$ ionic bond forms upon retraction from the surface (point 4), even at the same height $Z=5.9 \AA$. This hysteresis of the ionic system produces a nonconservative force on the spring and the energy dissipation in the AFM model [24].

The stability of the ionic configuration of the tip is also explained on the basis of the PESs. At $Z=6.4 \AA$, the ionic system at point 4 can return to that at point 1 since the magnitude of the barrier on the PES decreases. The ionic configuration returns to the initial one as the tip approaches and retracts from the surface; it is stable during the adhesion process. The depths of points 3 and 4 on the PESs become shallow as the tip is far from the surface, and the barrier on the PES vanishes at $Z=6.4 \AA$. Since the magnitudes of the depths of these minima correspond to the strength of the $\mathrm{Mg}-\mathrm{O}$ bond, these shallow minima mean that the $\mathrm{Mg}-\mathrm{O}$ bond weakens and brakes when the tip is far from the surface.

The magnitude of the barrier on the PES and the height dependence play key roles in the stability of the tip configuration during the adhesion process. The stability of


FIG. 5. Height dependence of the PES of the ionic system of the type 2 tip.

the tip relies on the ionic configuration of the tip and its PES during the adhesion process.

\section{B. Type 2 tip: unstable tip}

In the AFM model, the ionic configuration of the type 2 tip is irreversibly changed once the tip approaches the surface, as shown in Fig. 1(b). The type 2 tip becomes unstable during the adhesion process. Figure 5 shows the height dependence of the PES of the ionic system for the type 2 tip. The double minima and energy barrier for the PESs are similar to those observed for the ionic configuration for the type 1 tip; however, the barrier on the PES at $Z=6.8 \AA$ remains prominent. Once the minimum on the $\mathrm{PES}$ reaches point 3 at a $\mathrm{Mg}-\mathrm{O}$ separation of approximately $2 \AA$, the ionic system remains at points 4 and 5 even after retraction far from the surface owing to the barrier. Point 6 located at top of the barrier is energetically unstable, and the ionic system is never at this point and returns to point 1 . This situation is different from that observed for the type 1 tip. Once a $\mathrm{Mg}-\mathrm{O}$ bond forms between the tip and the surface, it does not break, even upon retraction of the tip, and the $\mathrm{Mg}$ and $\mathrm{O}$ ions never return to their original lattice sites. Such strong bonding between $\mathrm{Mg}$ and $\mathrm{O}$ ions lowers the energetic minimum at point 5 further and maintains the barrier on the PES, even when the tip is far from the surface.

The $\mathrm{Mg}-\mathrm{O}$ bond forms when the tip approaches the surface, in which the strength of the $\mathrm{Mg}-\mathrm{O}$ bond depends not only on the bond length but also the ionic arrangements around the $\mathrm{Mg}-\mathrm{O}$ bond. The ionic arrangements as shown as point 4 in Fig. 5 make the $\mathrm{Mg}-\mathrm{O}$ bond strong when the tip retracts from the surface, while in the case of point 4 in Fig. 4 of the type 1 tip, the $\mathrm{Mg}-\mathrm{O}$ bond becomes weak upon the retraction. It is currently not clear which part of those ionic arrangements contributes the strength of the $\mathrm{Mg}-\mathrm{O}$ bond, and this remains a challenge for further study.

According to the results obtained from the AFM model [24], once the $\mathrm{Mg}-\mathrm{O}$ bond forms, it becomes a string of ions connecting between tip and surface upon retraction, as shown in Fig. 1(b). The formation of the strings is due to the strong $\mathrm{Mg}-\mathrm{O}$ bond and kinetic energy of the ionic system at finite temperature $T=300 \mathrm{~K}$ in the AFM 


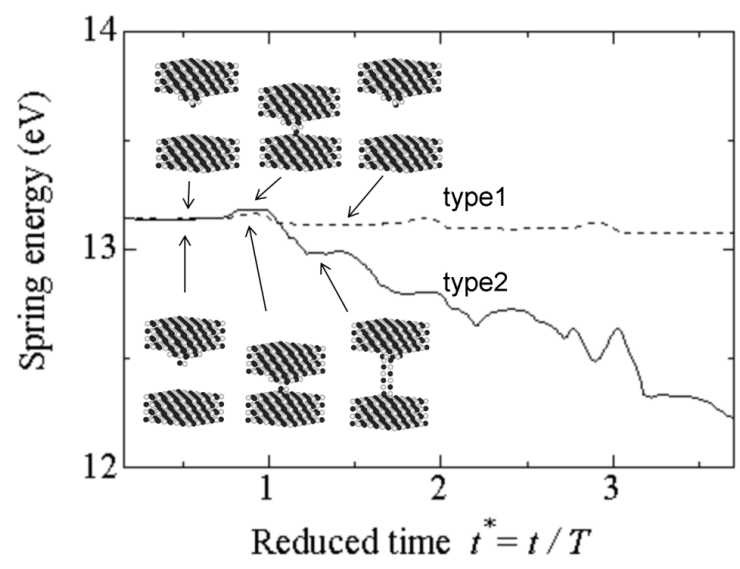

FIG. 6. Time evolutions of the energy of the spring in the AFM model using the coupling method shown in Fig. 1(a). The period time $T$ of the spring's oscillation is $6.76 \times 10^{-11} \mathrm{~s}$. Snapshots of the ionic systems are superimposed.

model. The finite temperature is expected to considerably affect the ionic configuration of tip and surface.

\section{Tip stability versus energy dissipation}

It is found from calculation using the AFM model that the magnitude of the dissipation per cycle increases for the type 2 tip, as shown in Fig. 6, although the initial configuration of the tip irreversibly changes to strings of ions. The amounts of the energy dissipation of the spring are about $0.02 \mathrm{eV} /$ cycle for type 1 and $0.2-0.3 \mathrm{eV} /$ cycle for type 2, where the same amplitudes of the oscillation are given initially for both types.

Figure 7 shows the force acting on the spring during approach and retraction of the spring, in which the force at approach is different from that at the retraction for both tip types. These nonconservative forces come from the hysteresis of the ionic system as explained in Sections III A and III B. The area between two force curves at approach and retraction corresponds to the quantity of energy dissipation per cycle of the spring oscillation. For the type $1 \mathrm{tip}$, once the $\mathrm{Mg}-\mathrm{O}$ bond forms [points 3 and 4 in Fig. $7(\mathrm{a})$ ], the attractive force is applied on the spring. For the type 2 tip, an attractive force (sometimes repulsive force) appears over the wide range of the spring position upon the retraction, which gives rise to a large area in the force curve and the large dissipation. Once the string forms between the tip and surface, it provides attractive force on the spring and it is stretched $[1 \rightarrow 2$ in Fig. $7(\mathrm{~b})]$ by spring's force in the AFM model upon the retraction. When the tensile force in the string increases to around $-0.05 \mathrm{eV} / \AA$, ions are supplied from the tip to the string and a longer string appears $[2 \rightarrow 3$ in Fig. $7(\mathrm{~b})]$. This new string with additional ions is stretched again as the retraction continues. The process of stretching and growth of the string occurs four times during the retraction of the spring in the present case, as shown in Fig. 7(b). This process upon retraction provides the attractive force on the spring over a wide range of spring positions and results in the large dissipation. The type 2 tip shows instability; however, it provides significant dissipation.

In contrast, for the type 1 tip, there is only one oppor-

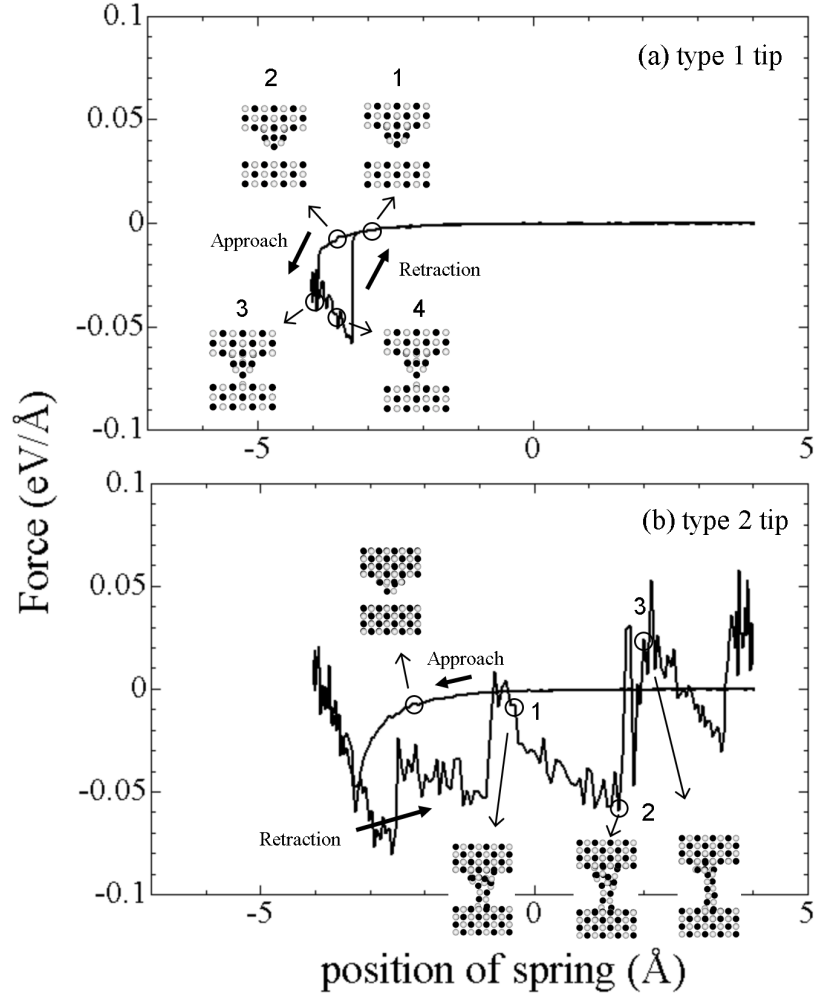

FIG. 7. Forces on the spring in the AFM model. The snapshots of the ionic system at the corresponding points (open circles) are superimposed.

tunity to contribute the attractive force in the force curve upon the retraction, which results in a smaller amount of dissipation than that for type 2 . The configuration of the type 1 tip remains even after many cycles of oscillation; however, its dissipation is comparably small.

\section{CONCLUSION}

The stability of a tip-namely, whether its ionic configuration is reconstructed or irreversibly changed in the process of adhesion - depends on the nature of the ionic configuration of the tip. For both tip types, when the tip approaches the surface, the $\mathrm{Mg}$ ion of the tip bonds to the $\mathrm{O}$ ion of the surface. In the type 1 atomic configuration, the $\mathrm{Mg}-\mathrm{O}$ bond breaks when the tip is retracted from the surface, which is reflected by the decrease in the barrier in the PES. In this case, since the $\mathrm{Mg}-\mathrm{O}$ bond is weak, the $\mathrm{Mg}$ ion can return to the original lattice of the tip, and the tip is reconstructed upon retraction. In contrast, in the type 2 atomic configuration, since the $\mathrm{Mg}-\mathrm{O}$ bond is strong, the minimum point on the PES at a separation equal to the $\mathrm{Mg}-\mathrm{O}$ bond length maintains its depth, and the barrier remains prominent, even upon the retraction. The $\mathrm{Mg}-\mathrm{O}$ bond remains intact as the tip retracts from the surface, and the ionic configuration of the tip and surface is irreversibly altered. We show that the strength of the $\mathrm{Mg}-\mathrm{O}$ bond largely determines the magnitude of the barrier on the PESs, and whether tip ions will return to their original configuration or undergo an irreversible change. However, the part of the ionic arrangements of the tip and surface that contributes to the strength of 
the $\mathrm{Mg}-\mathrm{O}$ bond remains to be clearly identified, which is a challenge for further studies on tip stability. It is shown from the AFM model coupling the ionic system to the spring oscillation that energy dissipation is comparably small for the stable type 1 tip, while for the unstable type 2 tip it provides significant dissipation.

\section{ACKNOWLEDGMENTS}

This study was supported by the Japan Society for the Promotion of Science, JSPS KAKENHI Grant No.25390081. This research has been performed under the Inter-University Cooperative Research Program of the Center for Computational Materials Science, Institute for Materials Research, Tohoku University (Proposal No. 17K0038).
[1] S. Morita, R. Wiesendanger, and E. Meyer (Eds.), Noncontact Atomic Force Microscopy (Springer, Berlin, Heidelberg, 2002).

[2] B. Anczykowski, B. Gotsmann, H. Fuchs, J. P. Cleveland, and V. B. Elings, Appl. Surf. Sci. 140, 376 (1999).

[3] R. Bennewitz, A. S. Foster, L. N. Kantorovich, M. Bammerlin, C. Loppacher, S. Schär, M. Guggisberg, E. Meyer, and A. L. Shluger, Phys. Rev. B 62, 2074 (2000).

[4] C. Loppacher, R. Bennewitz, O. Pfeiffer, M. Guggisberg, M. Bammerlin, S. Schär, V. Barwich, A. Baratoff, and E. Meyer, Phys. Rev. B 62, 13674 (2000).

[5] T. Fukuma, K. Umeda, K. Kobayashi, H. Yamada, and K. Matsushige, Jpn. J. Appl. Phys. 41, 4903 (2002).

[6] H. J. Hug and A. Baratoff, in: Noncontact Atomic Force Microscopy, edited by S. Morita, R. Wiesendanger, and E. Meyer (Springer, Berlin, Heidelberg, 2002) p. 395.

[7] M. Ashino, R. Wiesendanger, A. N. Khlobystov, S. Berber, and D. Tomanek, Phys. Rev. Lett. 102, 195503 (2009).

[8] Y. Naito, Y. J. Li, H. Nomura, M. Kageshima, and Y. Sugawara, J. Phys. Soc. Jpn. 79, 013601 (2010).

[9] K. Iwata, S. Yamazaki, Y. Tani, and Y. Sugimoto, Appl. Phys. Express 6, 055201 (2013).

[10] M. Gauthier and M. Tsukada, Phys. Rev. B 60, 11716 (1999).

[11] M. Gauthier, R. Paerez, T. Arai, M. Tomitori, and M. Tsukada, Phys. Rev. Lett. 89, 146104 (2002).

[12] L. N. Kantorovich, Phys. Rev. B 64, 245409 (2001).

[13] T. Trevethan and L. N. Kantorovich, Phys. Rev. B 70, 115411 (2004).

[14] T. Trevethan and L. N. Kantorovich, Nanotechnology 15 S44 (2004).

[15] A. L. Shluger, L. N. Kantorovich, A. I. Livshits, and M.
J. Gillan, Phys. Rev. B 56, 15332 (1997).

[16] G. Cross, A. Schirmeisen, A. Stalder, P. Grütter, M. Tschudy, and U. Dürig, Phys. Rev. Lett. 80, 4685 (1998).

[17] N. Sasaki and M. Tsukada, Jpn. J. Appl. Phys. 39, L1334 (2000).

[18] T. Trevethan and L. Kantorovich, Nanotechnology 16, S79 (2005).

[19] L. N. Kantorovich and T. Trevethan, Phys. Rev. Lett. 93, 236102 (2004).

[20] T. Trevethan and L. Kantorovich, Nanotechnology 17, S205 (2006).

[21] N. Oyabu, P. Pou, Y. Sugimoto, P. Jelinek, M. Abe, S. Morita, R. Pérez, and Ó. Custance, Phys. Rev. Lett. 96, 106101 (2006).

[22] S. Kawai, F. F. Canova, T. Glatzel, A. S. Foster, and E. Meyer, Phys. Rev. B 84, 115415 (2011).

[23] F. F. Canova and A. S. Foster, Nanotechnology 22, 045702 (2011).

[24] Y. Senda, J. Blomqvist, and R. Nieminen, Appl. Sci. Converg. Technol. 26, 6 (2017).

[25] Y. Senda, N. Imahashi, S. Shimamura, J. Blomqvist, and R. M. Nieminen, e-J. Surf. Sci. Nanotech. 12, 339 (2014).

[26] Y. Senda, J. Blomqvist, and R. Nieminen, J. Phys.: Condes. Matter 28, 375001 (2016).

[27] C. Barth and C. R. Henry, Phys. Rev. Lett. 91, 196102 (2003).

[28] M. L. Trouwborst, E. H. Huisman, F. L. Bakker, S. J. van der Molen, and B. J. van Wees, Phys. Rev. Lett. 100, 175502 (2008).

[29] A. I. Livshits, A. L. Shluger, A. L. Rohl, and A. S. Foster, Phys. Rev. B 59, 2436 (1999).

[30] M. Matsui, J. Chem. Phys. 91, 489 (1989). 